\title{
Gender Differences in Nighttime Sleep Patterns and Variability Across the Adult Lifespan: A Global-Scale Wearables Study
}

\author{
Jonasdottir, Sigga Svala; Minor, Kelton; Lehmann, Sune
}

Published in:

Sleep

Link to article, DOI:

10.1093/sleep/zsaa169

Publication date:

2021

Document Version

Peer reviewed version

Link back to DTU Orbit

Citation (APA):

Jonasdottir, S. S., Minor, K., \& Lehmann, S. (2021). Gender Differences in Nighttime Sleep Patterns and Variability Across the Adult Lifespan: A Global-Scale Wearables Study. Sleep, 44(2), [zsaa169].

https://doi.org/10.1093/sleep/zsaa169

\section{General rights}

Copyright and moral rights for the publications made accessible in the public portal are retained by the authors and/or other copyright owners and it is a condition of accessing publications that users recognise and abide by the legal requirements associated with these rights.

- Users may download and print one copy of any publication from the public portal for the purpose of private study or research.

- You may not further distribute the material or use it for any profit-making activity or commercial gain

- You may freely distribute the URL identifying the publication in the public portal

If you believe that this document breaches copyright please contact us providing details, and we will remove access to the work immediately and investigate your claim. 


\title{
Gender differences in nighttime sleep patterns and variability across the adult lifespan: a global-scale wearables study
}

\author{
Sigga Svala Jonasdottir ${ }^{1}$, Kelton Minor ${ }^{2, \bullet}$ and Sune Lehmann ${ }^{1,2, *}$ \\ ${ }^{1}$ Department of Applied Mathematics and Computer Science, Technical University of Denmark, Lyngby, Denmark and \\ ${ }^{2}$ Copenhagen Center for Social Data Science, University of Copenhagen, Copenhagen, Denmark \\ ${ }^{*}$ Corresponding author. Sune Lehmann, Technical University of Denmark, Anker Engelunds Vej 1, Building 101A, 2800 Kongens Lyngby, Denmark. \\ Email: sljo@dtu.dk.
}

\begin{abstract}
Study Objectives: Previous research on sleep patterns across the lifespan have largely been limited to self-report measures and constrained to certain geographic regions. Using a global sleep dataset of in situ observations from wearable activity trackers, we examine how sleep duration, timing, misalignment, and variability develop with age and vary by gender and BMI for nonshift workers.

Methods: We analyze 11.14 million nights from 69,650 adult nonshift workers aged 19-67 from 47 countries. We use mixed effects models to examine age-related trends in naturalistic sleep patterns and assess gender and BMI differences in these trends while controlling for user and country-level variation.

Results: Our results confirm that sleep duration decreases, the prevalence of nighttime awakenings increases, while sleep onset and offset advance to become earlier with age. Although men tend to sleep less than women across the lifespan, nighttime awakenings are more prevalent for women, with the greatest disparity found from early to middle adulthood, a life stage associated with child-rearing. Sleep onset and duration variability are nearly fixed across the lifespan with higher values on weekends than weekdays. Sleep offset variability declines relatively rapidly through early adulthood until age 35-39, then plateaus on weekdays, but continues to decrease on weekends. The weekend-weekday contrast in sleep patterns changes as people age with small to negligible differences between genders.
\end{abstract}

Conclusions: A massive dataset generated by pervasive consumer wearable devices confirms age-related changes in sleep and affirms that there are both persistent and life-stage dependent differences in sleep patterns between genders.

\section{Statement of Significance \\ A global dataset from wearable devices enables a detailed understanding of age-related tendencies in sleep patterns, controlling for country-level and within-individual variation. During early adulthood, we find elevated levels of variability in sleep offset and duration along with high levels of weekend-weekday misalignment, suggesting that mismatches between internal timing and external demands are pervasive during this phase of human development. In older adulthood, reduced sleep duration and increased sleep disturbances may either contribute to, or correlate with, further age-related decline. Gender gaps in average sleep duration, timing and nighttime awakenings are apparent, despite considerable heterogeneity in circadian preferences. Information about parenting mobile application usage can be paired with big data from wearable devices to explore lifestage gender inequality in sleep quality. Further research on person-centered behavioral interventions that promote regular sleep-wake cycles are needed.}

Key words: sleep; big data; aging; gender; sleep variability; sleep misalignment; sleep timing and duration

Submitted: 6 January, 2020; Revised: 4 August, 2020

(c) Sleep Research Society 2020. Published by Oxford University Press on behalf of the Sleep Research Society.

All rights reserved. For permissions, please e-mail journals.permissions@oup.com. 


\section{Introduction}

Sufficient sleep is fundamental to healthy human functioning. Brief, irregular and/or disturbed sleep are risk factors for infectious disease, cardiovascular disease, depression, and all-cause mortality [1-3]. Similar to other physiological functions, sleep patterns vary between people across the human population and change within individuals over the lifespan [4-6]. Meta-analyses and cross-sectional research provide convergent evidence that the ability to initiate and maintain sleep declines as people age, independent of factors such as medical co-morbidities and medication use $[7,8]$. However, large-scale in situ data on changes in sleep patterns across the lifespan remain scarce and geographically constrained.

Age-associated changes in sleep include both decreases in total time asleep, deep (slow wave) sleep, and rapid eye movement sleep, as well as increases in sleep latency, time awake after sleep onset, stage 1 and stage 2 sleep [7, 9, 10]. Increases in nighttime awakenings with age suggest a decline in the buildup of homeostatic sleep pressure [11]. Evidence that healthy older adults exhibit less objective and subjective sleepiness after selective slow wave sleep deprivation indicates that sleep need may decline as adults age [12], while other research has shown that reduced sleep still negatively impacts cognitive performance, irrespective of age $[13,14]$. A recent review concluded that while there is still no consensus, the current body of evidence largely supports the hypothesis that older adults have an impaired ability to generate sleep rather than a reduced sleep need [15]. Hence, additional insight is needed to characterize structural changes in sleep patterns over the lifespan and to better understand the underlying drivers.

Aside from changes in the duration and composition of sleep, adult aging is further characterized by changes in circadian regulatory processes, with phase advances and diminished amplitudes in daily core body temperature, melatonin, and cortisol rhythms [11]. These changes are associated with, and often proxied by, aging-related advances in sleep timing after adolescence, with individuals going to bed and waking up earlier with increasing age [16-20].

Previous research has established the importance of measuring sleep timing separately on both free and work days, since weekly social schedules constrain daily rhythms and can induce a misalignment with biological time [21].

In a series of large scale survey-based studies conducted with participants from four countries (Germany, Switzerland, the Netherlands, and Austria), both the difference between free and work day sleep duration and midsleep timing (social jet lag) were shown to decline with age $[22,23]$. Importantly, the authors did not report how underlying differences in sleep onset and offset timing between work and free days may contribute to observed developments in misalignment. Since people exert more practical influence over the beginning and end of their sleep period compared with midsleep, an expectation of how onset and offset change on weekdays and weekends with age would be useful.

Moreover, while a recent study found that inter-individual variability in midsleep timing declines with age [24], far less is known about how intra-individual variability in sleep timing and duration within work and free day periods changes across the life course [25-27]. In situ sleep data collected over an extended period is needed to inform expectations about age-related developments in sleep timing, misalignment, and variability.

Prior research has established that the extent of adult age-related changes in sleep patterns is highly moderated by gender, with women reaching both puberty and their peak eveningness earlier than men in young adulthood and sleeping longer than men until age 50-60, a period of adulthood that coincides with menopause [23, 28-30]. Well-controlled laboratory studies have found that women exhibit phase advanced core body temperature and melatonin rhythms, as well as a shorter intrinsic circadian period compared with men [31, 32]. While some larger sleep surveys have found that females are more likely to be morning types compared with males from the end of adolescence to late adulthood [17, 33], others have found no apparent gender differences [34,35] and one nationally representative study found the opposite trend [36]. Notably, these cross-sectional studies were conducted within different countries using slightly different methodologies, highlighting the need for an integrated global assessment of potential genderrelated differences in sleep. Beyond long-term changes in sleep patterns, events occurring during certain stages of adult development can impact women and men differently. Pregnancy and the postpartum period are associated with dynamic physiological changes and behavioral demands known to disturb sleep quality for women, although limited in situ evidence exists comparing sleep disturbances for both women and men during young child-rearing [37, 38].

Despite revealing salient age-related changes in sleep patterns, there are a number of areas where previous research can be extended and improved. Early lifespan meta-analyses favored data from predominantly Western countries, averaged across different sleep assessment methodologies and depended extensively on short-term polysomnography recordings that may have disrupted participants' habitual sleep cycles [7, 9]. By comparison, cross-sectional sleep survey research has primarily relied on subjective measures prone to self-report, recall, and rounding biases [17, 24, 39, 40]. Moreover, large-scale sleep surveys typically ask for single estimates of work and free day sleep onset and offset times, and thus do not enable measurement of the intra-individual variability of sleep patterns during work and free day periods [26]. To address these limitations, recent research has drawn upon behavioral data from mobile phones and self-tracking apps to infer the dynamics of human activity and sleep in everyday contexts across large populations of device users [41-47]. Differing from mobile phones, the activity trackers employed in the current study were worn by users, enabling closely coupled measurements of human sleep patterns. Similar to wrist-actigraphs, wearable devices can automatically monitor sleep measurements in situ over extended periods of use, making it possible to study both average and time-varying sleep patterns in daily contexts [20,48,49]. Drawing on a dataset of objective sleep measurements and mobile application use statistics from a large sample of $n=69,650$ wearable users over multiple years across 47 countries, we investigate the following questions:

1. After controlling for country-level and individual-level variation with mixed effects models, does global data from consumer wristband devices confirm gender differences in age-related changes in sleep duration, timing, and circadian misalignment? 
2. How does intra-individual variability in sleep duration and timing develop across the life course separately on weekdays and weekends, adjusting for country-level variation?

3. Does child-rearing - as proxied by parenting mobile application use-predict life-stage gender differences in nighttime sleep disturbances?

The present study differs from prior self-report studies, most of which featured self-report data from single countries or regions, and-at the time of writing-represents the most geographically extensive analysis of age-associated changes in sleep using consistent, objectively recorded measures of sleep duration, timing, misalignment, and variability.

\section{Methods}

\section{Data and demographics}

The anonymized data set used in this study consists of sleep observations collected using smart wristbands from 2015 to 2018 (see the section Data collection below for full details). In total, we analyze 11.14 million nights of sleep observations arising from 69,650 adult nonshift workers, about a third of them women. In Table 1, we show the number of individuals and nights broken out by the demographic variables age group, gender, and BMI category and in Supplementary Table S1 lists all the countries in our sample and ratio of users residing there. We compare age statistics (median age) in our sample to information provided by the United Nation Population Division (UN) [50] for the five countries with the most users in the dataset in Supplementary Table S2. The median values in our sample and the overall population correspond well: users from Japan are slightly younger (by 1 year) while those from Taiwan and the United Kingdom match their respective reference populations. By comparison, users from Germany are younger (by 7 years) as well as those from Russia (by 5 years). We also compare age standardized BMI statistics of the study sample to population estimates provided by the World Health Organization (WHO) in Supplementary Table
S3 [51, 52]. We find both men and women from all countries fall within or near the $95 \%$ confidence intervals (CIs) of the WHO reference values. Women from the UK fall 0.5 points above the $95 \% \mathrm{CI}$ and women from Japan average 0.5 points below the $95 \%$ CI reference range.

\section{Sleep duration, timing, and variability outcomes}

We use nine sleep metrics to assess how sleep patterns change across the lifespan. Sleep duration specifies the total recorded time a person spent asleep during a given night. To quantify sleep timing, we use sleep onset (the registered point in time when a person fell asleep) and sleep offset (the recorded time when a person woke up). We measure the misalignment between an individual's internal biological clock and external social clock by applying a variant of the formula used to compute social jetlag [22]. Specifically, instead of calculating social jetlag for midsleep (see Supplementary Figure S8 for a comparison) we estimate weekend-weekday differences in sleep onset and offset. The weekend-weekday misalignment of sleep timing can lead to the loss of sleep duration on weekdays and partial compensation on weekends, which is quantified by estimating the weekendweekday sleep duration difference. These metrics are calculated for each week of data collection, resulting in weekly repeated measurements for each user, which are then aggregated to produce user-level averages. We also study the variability of sleep onset, offset, and duration in order to estimate the regularity of people's sleep timing and duration. We quantify intra-individual variability as the standard deviation of a person's corresponding measurements for each sleep outcome, and compute this separately for weekends and weekdays [53].

The individual-level covariates for this study are gender (female/male) and BMI categories (underweight/normal weight/ overweight/obese) which were labelled according to the World Health Organization classification [54, 55].

Additionally, we also include temporal variables for day category (weekday/weekend) to account for likely differences in the social structure over the course of the week. Since we do not

Table 1. Overview of the data set with a focus on demographics: age, gender, and BMI

\begin{tabular}{|c|c|c|c|c|c|c|}
\hline \multicolumn{4}{|c|}{ \# of adult users in sample } & \multicolumn{3}{|c|}{ \# of night sleep observations } \\
\hline & All & Male & Female & All & Male & Female \\
\hline Total & 69,650 & 47,656 & 21,993 & $11,144,539$ & $7,673,495$ & $3,471,044$ \\
\hline \multicolumn{7}{|l|}{ Age groups } \\
\hline $19-24$ & 5,466 & 3,745 & 1,721 & 579,315 & 383,761 & 195,554 \\
\hline $25-29$ & 8,976 & 5,813 & 3.163 & $1,105,037$ & 698,471 & 406,566 \\
\hline $30-34$ & 11,224 & 7,414 & 3,810 & $1,559,445$ & $1,022,033$ & 537,412 \\
\hline $35-39$ & 9,796 & 6,584 & 3,212 & $1,520,749$ & $1,024,874$ & 495,875 \\
\hline $40-44$ & 9,315 & 6,435 & 2,880 & $1,591,014$ & $1,092,829$ & 498,185 \\
\hline $45-49$ & 9,934 & 6,994 & 2,940 & $1,844,717$ & $1,308,604$ & 536,113 \\
\hline $50-54$ & 7,164 & 5,059 & 2,105 & $1,395,210$ & 999,842 & 395,368 \\
\hline $55-59$ & 4,445 & 3,180 & 1,265 & 879,907 & 646,560 & 233,347 \\
\hline $60-67$ & 3,330 & 2,433 & 897 & 669,145 & 496,521 & 172,624 \\
\hline \multicolumn{7}{|l|}{ BMI categories } \\
\hline Underweight & 2,272 & 1,197 & 1,075 & 350,954 & 173,220 & 177,734 \\
\hline Normal weight & 34,063 & 22,101 & 11,962 & $5,773,876$ & $3,843,837$ & $1,930,039$ \\
\hline Overweight & 22,936 & 17,371 & 5,565 & $3,558,454$ & $2,687,277$ & 871,177 \\
\hline Obese & 10,379 & 6,988 & 3,391 & $1,461,255$ & 969,161 & 492,094 \\
\hline
\end{tabular}

The table provides statistics for both the number of adults in the sample, as well as the number of nights analyzed. Note that the data set contains more men than women and more people within the normal weight range BMI category. 
directly observe schedules, we assume the likelihood of workdays is highest on weekdays and work-free days is highest on weekends, similar to others $[20,24]$.

\section{Data modeling}

We analyzed the data set using R Version 3.5.1 [56]. Given the longitudinal and hierarchical structure of the data with repeated measurements within users, and users nested within their country of residence, observations are likely highly correlated on both levels (country and user). To account for this dependence within the data set, we adopt a mixed effects modeling framework [57]. Mixed effects models allow us to control for user and country-level variation while examining age-related trends in sleep patterns and assessing the influence of demographic factors. Concretely, the model can be specified in matrix form as

$y=X \beta+Z u+\varepsilon, \quad$ with $u \sim N_{q}(0, G)$ and $\varepsilon \sim N_{n}(0, R)$,

with $\beta$ representing the fixed effects parameters, $u$ representing the random effects, $X$ representing the $n * p$ design matrix for the fixed-effects parameters, and $Z$ the $n * q$ design matrix describing the random effects. The models for weekdayweekend differences and sleep variability are defined without user random effects since these measures were computed for each user as single values. We use the lmerTest $\mathrm{R}$ package, the lmer function to fit the data set and apply Satterthwaite's degrees of freedom method to estimate the $p$-values for the significance of fixed factors [58, 59].

We center the age variable around its mean to help improve interpretability, prevent multicollinearity, and lower the scale of the variables to accommodate the inclusion of age squared in the model.

\section{Data collection}

Data were collected from 2015 to 2018 via Sony SmartBand (SmartBand Talk [SWR30] and SmartBand 2 [SWR12]), designed to track physical activity and sleep behavior. The waterproof wristbands use proprietary, internally validated algorithms based on movement registered by an internal accelerometer to estimate sleeping and waking states in 1-min epochs. When connecting the wristband at first, users received visual instruction on how and where (wrist) to place the device and were advised to wear it on their dominant side. All wearable data included in this study were wirelessly transmitted via Bluetooth to an accompanying mobile phone application, which also independently registered user mobile application usage statistics. Similar to many other wearable devices and wrist actigraphs, the devices used in the present study detect sleep timing and total sleep time but do not detect time in bed, preventing the further study of age-related changes in sleep latency and sleep efficiency. Moreover, although the armbands have been validated internally within SONY, we note that the wristbands have not been publicly validated using the gold standard of polysomnography as recommended inn the Sleep Research Society Workshop on wearable devices for the measurement of sleep [60]. The wristbands employed in this study have been shown to produce wake and sleep states that converge with objective measures of user mobile phone use patterns [46]. However, this global dataset offers unique methodological advantages; scale, longitudinal coverage, and ecologically valid observations. By using it, we follow a growing trend of utilizing commercial devices in sleep research to study sleep behavior in naturalistic settings at large scales [39, 61, 62]. Further, we have performed an extensive comparison of the findings here with multiple independent global sleep datasets. We find that this worldwide dataset externally converges with country-level sleep measures from separate large-scale datasets, demonstrates consistency over the period of observation and replicates age-related sleep trends from previously published self-report studies, including changes in sleep duration and timing. These full comparisons are presented in the Supplementary Information Sections Comparison of countrylevel statistics to other publications and Consistency over time and Results. Further, the wristbands employed in this study have been shown to produce wake and sleep states that converge with objective measures of user mobile phone use patterns [46].

Study participants consist of anonymized users who consented to share their data for research purposes. Age group, BMI category, gender, and country of residence were preprocessed from self-reported demographic information. All data analyses were carried out in accordance with the EU's General Data Protection Regulation 2016/679 (GDPR) and the regulations set out by the Danish Data Protection Agency. The GDPR describes regulations for data protection and privacy in the European Union and the European Economic Area. It also addresses the transfer of personal data outside the EU and EEA areas.

\section{Data processing and inclusion criteria}

To reduce the risk of including sleep observations from those suffering from insomnia, artificially shortened sleep observations due to users ceasing wristband use in the middle of the resting period, observations from nightshift workers or any other possible problems, outliers from the sleep data were removed by applying inclusion filters to sleep duration, onset, and offset. We adopt standard filters for sleep duration $(3<$ duration $<13)$, matching those applied by Roenneberg et al. [23]. These filters are more inclusive (by $2 \mathrm{~h}$ ) than those used by Walch et al. [45] and Althoff [62] $(4<$ duration < 12). Furthermore, we apply the following conservative sleep timing filters. First, we remove all sleep observations with onset or offset times greater than one and a half standard deviations away from the sample average computed separately for weekdays and weekends and obtain the following time filters:

- 20:24 $\leq$ onset weekends $\leq 04: 52$

- 20:28 $\leq$ onset weekdays $\leq 03: 59$

- 03:59 $\leq$ offset weekends $\leq 12: 52$

- 03:21 $\leq$ offset weekdays $\leq 11: 25$

This results in the removal of $12 \%$ of sleep observations yielding a final dataset consisting of 11.14 million nights from 69,650 users. The full data preprocessing procedure is described in the section Data Filtering in the Supplementary Information.

To help ensure that sleep estimates are representative of typical sleeping behavior, we further require all participants to have a minimum threshold of sleep observations. Specifically, each user must have sleep observations extending over a minimum period of 4 weeks, with at least 1 weekday and weekend night per week, amounting to a minimum 8 nights per user 
(median 87 nights per user). We also limit our analysis to adults 19-67 years of age due to limited across-country data for older age groups. Each user is assigned a country of residence, defined as the country in which the majority of their sleep entries occur.

\section{Sociocultural variation}

This article focuses on how sleep duration, timing, misalignment, and variability develop with age and how other demographic factors such as gender and BMI may affect these trends. Hence, it is important to note that users in the sample reside across a wide range of countries around the world. Breaking the data set out by country of residence yields cohorts from 47 distinct countries with at least 150 users in each country. Supplementary Table S1 lists out all of the countries and percentage of users residing in each country, as well as the ratio of male users within each country. Table 2 shows the development of sleep onset and duration with age for men split up by the top 5 countries with the most users. It is evident that there is substantial heterogeneity in the amount and timing of sleep obtained between countries. The summary statistics reveal, consistent with the literature, that there are indeed large disparities in sleep patterns across cultures [20, 45, 63, 64]. Since the focus of the present research is to assess and identify age- and gender-related changes, we control for these baseline countrylevel differences through our mixed effects modeling framework described in Data modeling.

\section{Results}

\section{Sleep timing and duration over the lifespan}

In order to summarize the development of sleep onset, offset, and duration across the lifespan, we calculate each user's average value and then aggregate across our study sample by age, gender, and day type (weekday or weekend).
The resulting curves for sleep onset, offset, and duration are shown in Figures 1-3, D.

\section{Development of onset}

The main panel on Figure 1 shows that, overall, sleep onset becomes earlier as people age and that people tend to go to bed later on weekends (indicated by lighter colors); the difference between weekday and weekend is roughly constant for both men and women across all age-groups. There are large differences in mean onset time between men and women (more than $30 \mathrm{~min}$ for the 19-24 young adult age group), which progressively become smaller in magnitude across the lifespan, eventually falling out of the range of statistical significance for the 60-67 older adult age group. This eventual confluence of sleep onset is driven by a steeper age-related advance in sleep onset time for men than women. While the decline in sleep onset time is consistent for men, the rate of decrease in onset time for women nearly plateaus after the age 35-39 range. Even though the 95\% CIs for the mean are narrow, the actual distribution of sleep onset is quite broad, as shown in Figure 1, A-C, which shows the distribution of onset time for the 19-24 group, the 40-44 age group, and the 60-67 group. In order to directly visualize the progression of sleep onset timing between genders, in Figure 1, E (weekends) and Figure 1, F (weekdays), we display the difference of male/female onset from the average curve (genders weighted equally). The gender gap in onset time appears to persist until around age 40 , when the two curves begin to converge.

Figure 1, D plots the aggregated raw data from our sample; the displayed trends in sleep onset are confirmed by our modeling which adjusts for demographic covariates, and controls for individual and country baseline behavior. Sleep onset has a quadratic relationship with age $\left(p<2 * 10^{-16}\right.$, Supplementary Table S17). The model estimates a $29 \pm 0.20$ min difference between weekends and weekdays for women and $28 \pm 0.20 \mathrm{~min}$ difference for men (age group 40-44), with a

Table 2. Development of sleep onset and duration by age split up by the top five countries with the most users in the data set

\begin{tabular}{|c|c|c|c|c|c|}
\hline & Japan, $n=17,231(24.7 \%)$ & Germany, $n=7,140$ (10.3\%) & Russia, $n=5,095$ (7.3\%) & Taiwan, $n=5,028$ (7.2\%) & $\mathrm{UK}, n=3,900(5.6 \%)$ \\
\hline Age groups & \multicolumn{5}{|c|}{ Average sleep onset (hh:mm) } \\
\hline $19-24$ & $00: 53$ & 23:55 & 00:39 & 01:12 & $00: 24$ \\
\hline $25-29$ & $00: 44$ & $23: 52$ & $00: 29$ & $00: 59$ & 00:07 \\
\hline $30-34$ & $00: 41$ & $23: 40$ & $00: 21$ & 00:51 & $23: 52$ \\
\hline $35-39$ & $00: 27$ & $23: 36$ & 00:08 & $00: 42$ & $23: 46$ \\
\hline $40-44$ & $00: 21$ & 23:37 & 00:04 & $00: 32$ & 23:41 \\
\hline $45-49$ & 00:15 & 23:30 & 00:03 & $00: 30$ & $23: 45$ \\
\hline $50-54$ & $00: 06$ & 23:31 & $23: 58$ & $00: 16$ & 23:36 \\
\hline $55-59$ & $23: 54$ & $23: 27$ & $23: 55$ & $23: 52$ & $23: 45$ \\
\hline $60-67$ & $23: 42$ & $23: 26$ & $23: 50$ & $23: 50$ & $23: 42$ \\
\hline Age groups & \multicolumn{5}{|l|}{ Average sleep duration (h) } \\
\hline $19-24$ & 6.6 & 7.3 & 7.0 & 6.7 & 7.3 \\
\hline $25-29$ & 6.4 & 7.1 & 7.0 & 6.7 & 7.3 \\
\hline $30-34$ & 6.4 & 7.1 & 7.0 & 6.6 & 7.2 \\
\hline $35-39$ & 6.3 & 7.0 & 7.0 & 6.4 & 7.1 \\
\hline $40-44$ & 6.3 & 6.9 & 7.0 & 6.5 & 7.1 \\
\hline $45-49$ & 6.2 & 7.0 & 7.0 & 6.4 & 7.0 \\
\hline $50-54$ & 6.2 & 7.0 & 7.0 & 6.6 & 7.0 \\
\hline $55-59$ & 6.3 & 6.9 & 7.0 & 6.6 & 7.0 \\
\hline $60-67$ & 6.4 & 7.2 & 7.1 & 6.5 & 7.1 \\
\hline
\end{tabular}

Note there are strong differences between countries with a clear split between European and Asian countries. 

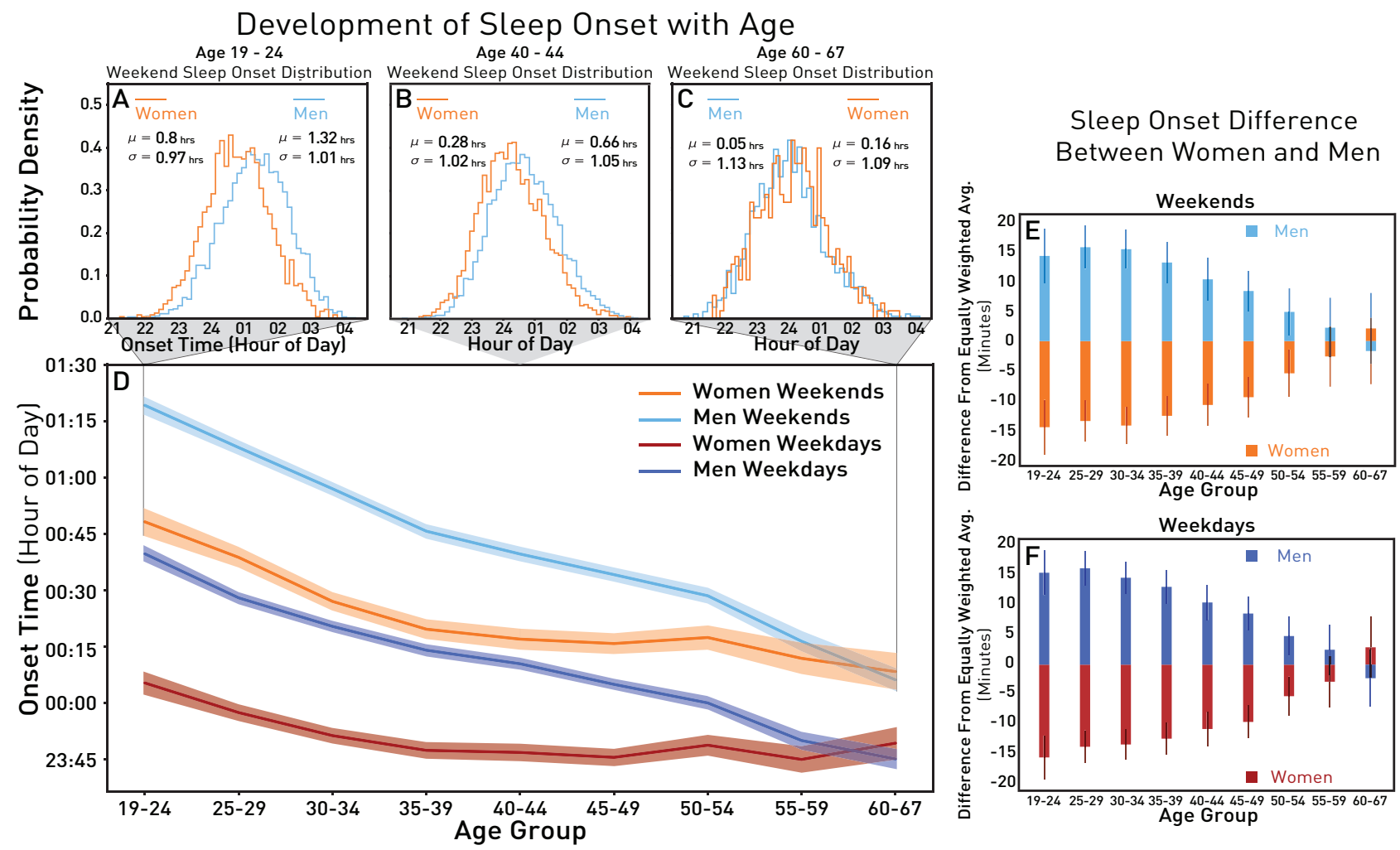

Figure 1. Distributions for sleep onset on weekends split up by gender for different age groups: (A) age 19-24, (B) age 40-44, and (C) age 60-67. The development of average sleep onset by age group split up by gender and day type (weekend/weekday). The red/orange colors correspond to women, light/dark blue colors correspond to men, darker colors represent weekdays and lighter colors signify weekends. The colored envelopes display $95 \%$ CIs around each age group mean (D). The equally weighted, between gender sleep onset difference by age group with $95 \%$ CI on weekends (E) and weekdays (F).

later average onset on weekends. When we consider the rate of decrease of sleep onset for men, we find the model results suggest an even steeper rate of decrease than the raw data (see Supplementary Figure S3 and Supplementary Table S17). Consequently, the difference between men and women at age 40-44 on weekdays is estimated to be $24 \pm 1.5 \mathrm{~min}$ based on the raw data but $15 \pm 1.5 \mathrm{~min}(95 \% \mathrm{CI})$ by the model. Furthermore, the model estimates the onset curves for men and women to intersect slightly earlier (within the 50-54 age range) than the raw data. From age 55 to 67 , the model indicates that men are expected to exhibit earlier onset than women. The mixed effects model indicates that there is a larger range of countrylevel random effect values for onset $(1.76 \mathrm{~h})$ than offset $(1.35 \mathrm{~h})$. This finding is in accordance with the results from a study conducted in 2014 using surveys and smartphone data: country of residence appears to exert a stronger influence on adult sleep onset than offset [45].

\section{Development of offset}

Turning to the development of sleep offset, Figure 2, D shows that the mean value of sleep offset mostly decreases with age, and people tend to wake up earlier as they get older. On weekdays, the curve is nearly flat for women between ages 45 and 59, but there is an increase for the age interval 60-67. Men consistently decrease in wake-up time with age except the slight increase from 60 to 67 on weekdays. The contrast between weekends and weekdays is nearly fixed across the lifespan with an hour difference resulting in later wake-up time on weekends. The curves on Figure 2, D show roughly the opposite behavior of what we observed for sleep onset (Figure 1, D), with the 95\% CI of the mean values for men and women overlapping until the middle of adulthood and thereafter diverging with men rising earlier than women. Thus, from age 19 to 39 women and men exhibit an average tendency to go to bed at different times yet wake up at similar times. The sleep offset curves for men and women diverge earlier on weekends (40-44) where the separation occurs one age group later (45-49) on weekdays. This can be seen even more clearly on Figure 2, E (weekends) and Figure 2, F (weekdays) which shows the difference of sleep offset by gender and age group from the equally weighted average of the curves for men and women.

Similar to the case of onset, the plotted mean offset values have small error bars (as indicated by the $95 \%$ confidence bands), while the actual distributions of sleep offset are quite broad. This is depicted in Figure 2, A-C, which shows the distribution of offset time for the 19-24 group, the 40-44 group, and the 60-67 group, respectively. We observe close agreement between the plots in Figure 2, D and the model results (Supplementary Table S19). Age, gender, and type of day are the most influential factors on wake-up time, which has a quadratic relationship with age $\left(p<10^{-16}\right.$, see Supplementary Table S19). For people aged 40-44, the model shows men to have the same sleep offset time as women, whereas on weekends they are expected to wake up $2.7 \pm 0.20$ min earlier (Supplementary Table S19). This is displayed in Figure 2, D, which shows that the sleep offset curves for men and women diverge earlier in the lifespan on weekends than weekdays. 


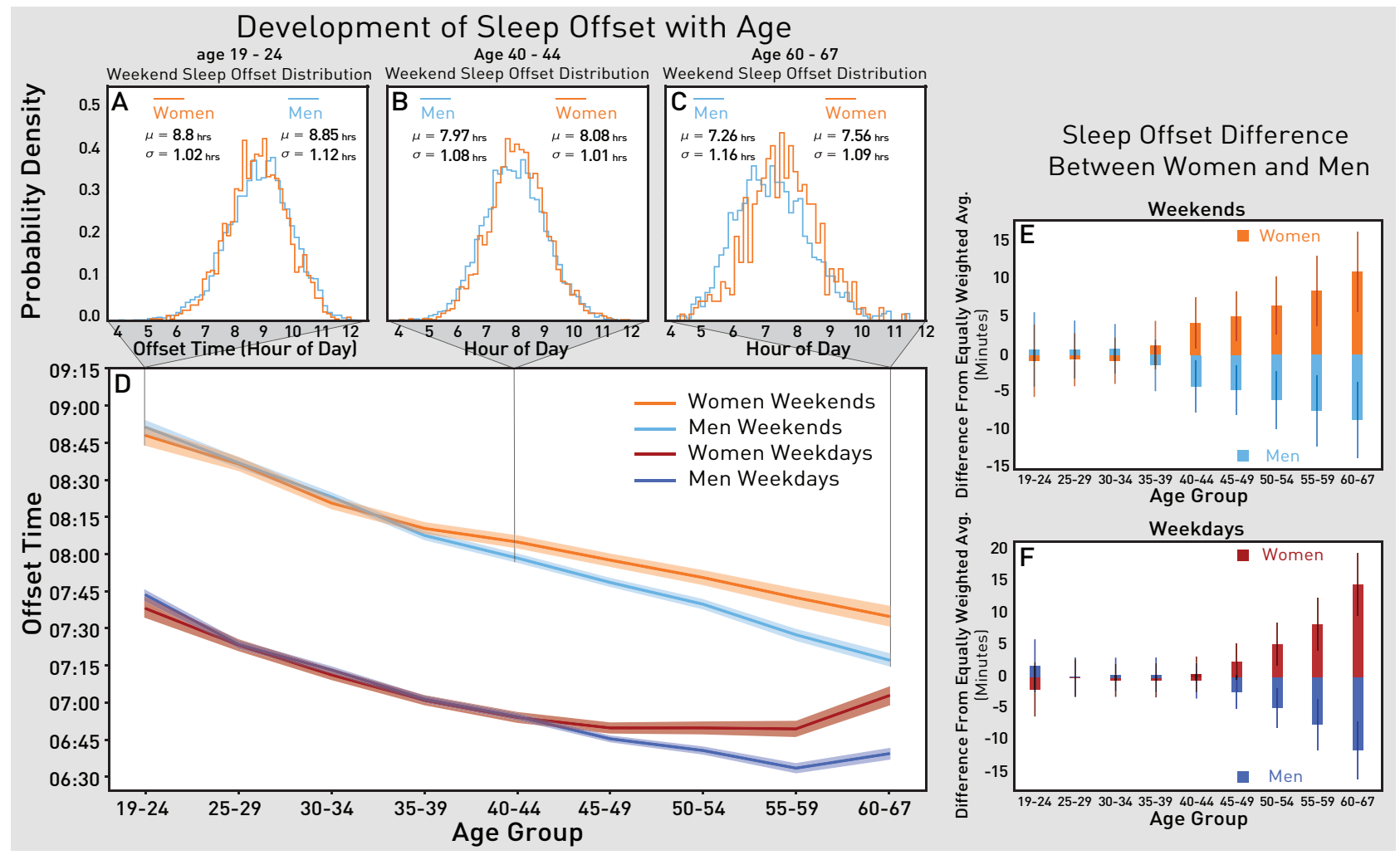

Figure 2. Distributions for sleep offset on weekends split up by gender for different age groups: (A) age 19-24, (B) age 40-44, and (C) age 60-67. The development of average sleep offset by age group split up by gender and day type (weekend/weekday). The red/orange colors correspond to women, light/dark blue colors correspond to men, darker colors represent weekdays and lighter colors signify weekends. The colored envelopes display $95 \%$ CIs around each age group mean (D). The equally weighted, between gender sleep offset difference by age group with $95 \%$ CI on weekends (E) and weekdays (F).

\section{Development of duration}

Figure 3, D shows that sleep duration tends to decrease across the lifespan. This development is nearly linear for weekends and less so on weekdays, with a small increase in duration for the oldest age group on weekdays. The difference between weekends and weekdays remains similar throughout the lifespan with a slightly smaller gap for men and women in the oldest group. This is highlighted in Figure 3, E (weekends) and Figure 3, F (weekdays) which show the difference of sleep duration by gender and age group from the equally weighted average of the curves for men and women. Although the average behavior shown in Figure 3, $\mathrm{D}$ exhibits statistically significant differences between men and women across different age groups, each aggregated group mean is derived from a broad range of underlying behavior as Figure 3, A-C shows the distributions of sleep duration for the 19-24 group, the 40-44 group, and the 60-67 group, respectively.

The mixed effects model for sleep duration, which controls for individual and country of residence variations, generally confirms the trends observed in the aggregated raw data plots visible in Figure 3 (for comparison of the raw data and model fit see Supplementary Figure S4). The weekend-weekday differences in duration are apparent in the model results but the magnitude of gender differences turn out smaller, due to different rates of change in sleep duration with age. Consequently, the curves for men and women come close to overlapping from age 55 to 67, see Supplementary Figure S4. Adjusting for BMI, the aggregated raw data estimates women at age 40-44 to sleep $23 \pm 1.7$ min longer than men, whereas the model estimates a difference of $11 \pm 1.0 \mathrm{~min}$ (95\% CI), see Supplementary Table S21 for estimates of fixed effects.

\section{Development of nighttime awakenings with age}

Having considered the progression of sleep onset, offset, and duration, we now assess how the prevalence of nighttime awakenings develops across adulthood. To quantify nighttime awakenings, we use wake after sleep onset (WASO) which refers to periods of wakefulness occurring after defined sleep onset and reflects sleep fragmentation [65]. For each registered night, WASO is the total time an individual is recorded awake (after defined sleep onset, but also occurring before defined sleep offset). Since sleep was recorded in 1-min epochs, only WASO measurements greater than $60 \mathrm{~s}$ were registered by the wristbands. We observe a large fraction of users with zero instances of WASO ( $85 \%$ of the users have a median WASO value of zero). This is in part because accelerometer-based fitness bands may underestimate sleep disruptions if individuals are awake but lying still in bed [66]. For that reason, our measure of nighttime awakenings may be conservative and correspond to relatively large sleep disruptions detectable by the embedded accelerometer. The percentage of users with nonzero median WASO is plotted by age group and gender in Figure 4. The percentage of individuals with nonzero median WASO increases with age; for the 19-24 age group, $4.5 \%$ of men and $9.7 \%$ of women have nonzero median WASO compared with $33.4 \%$ (men) and $35.6 \%$ (women) for the 60-67 age group. 

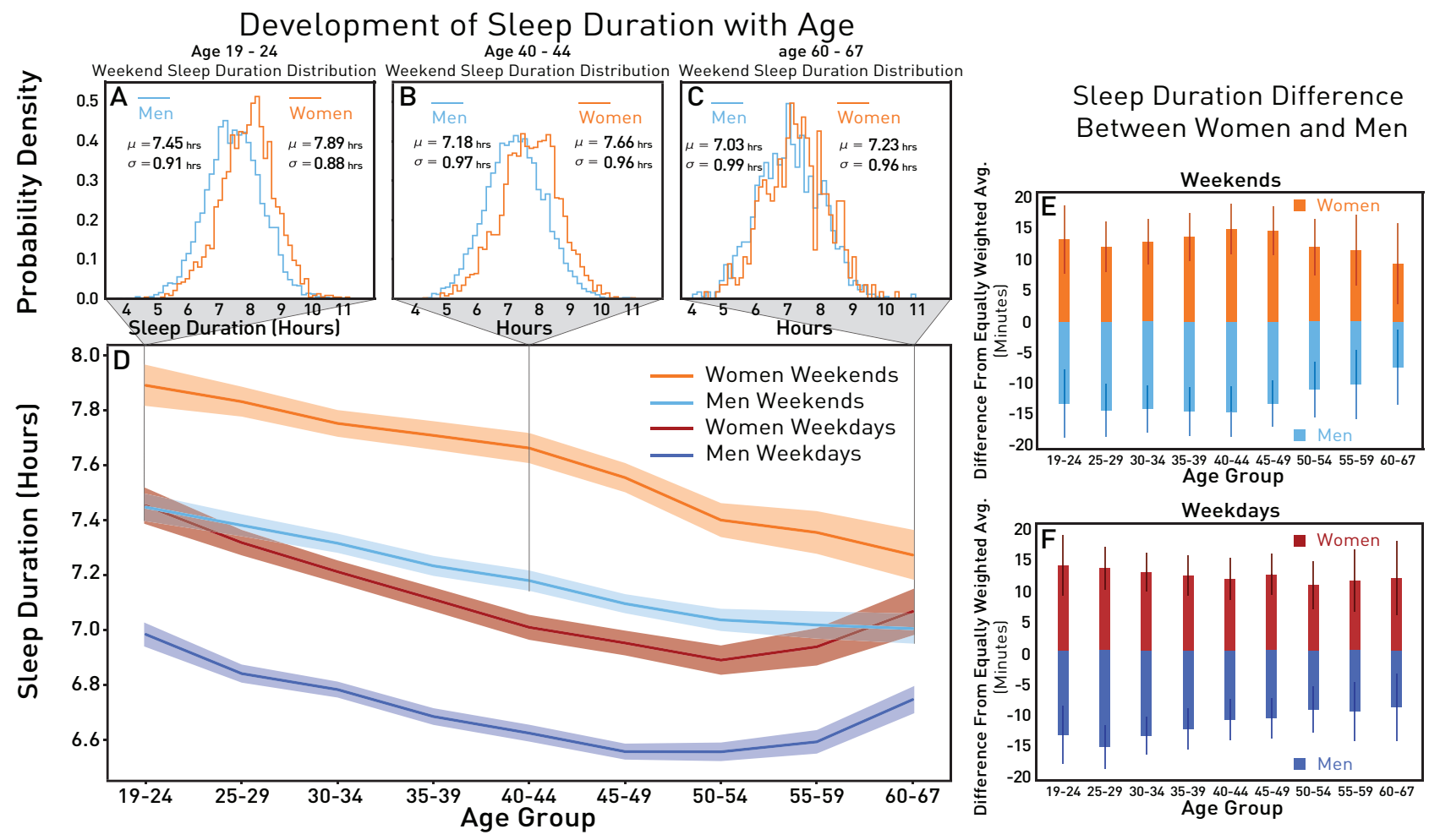

Figure 3. Distributions for sleep duration on weekends split up by gender for different age groups: (A) age 19-24, (B) age 40-44, and (C) age 60-67. The development of average sleep duration by age group with $95 \%$ CI split up by gender and day type (weekend/weekday). The red/orange colors correspond to women, light/dark blue colors correspond to men, darker colors represent weekdays and lighter colors signify weekends. The colored envelopes display $95 \%$ CIs around each age group mean (D). The equally weighted, between gender sleep duration difference by age group with $95 \%$ CI on weekends (E) and weekdays (F).

Figure 4 shows that a higher proportion of women have nonzero WASO medians than men from age 19 to 39. Specifically, $17.5 \%$ of women have nonzero WASO median values compared with $13.5 \%$ for men aged 19-39. The distribution of within individual median WASO differs significantly for men and women aged 19-39, estimated with two sample Kolmogorov-Smirnov (KS) statistics where $p=3.06 * 10^{-21}$. Interestingly, this same stage of life marks a biological window where childbirth, infant rearing and child caretaking are more likely [67]. As a post hoc analysis, we investigate the hypothesis that increased prevalence of nighttime awakenings during early adulthood may be linked to tending to infants and young children which exhibit irregular sleep patterns for the first 0-2 years of life [68]. For that reason, we analyze the age group 19-39, where the difference between the two curves in Figure 4 diverges between genders. As a proxy for information regarding parental status and infant-rearing, we reference aggregated app-context information. Specifically, we can anonymously identify users as probable parents if they have apps installed on their phones intended for parents with young children ("parent apps"). We describe how we identify apps as "parent apps" in the Supplementary Information: Identifying "parent apps." We find that women in the age range 19-39 with a parent app installed on their devices have a significantly different distribution of median (denoted M) WASO than age-matched women without the application on their phone (estimated with two sample KS statistics, $p=9.66 * 10^{-21}$ ), where $M_{\text {WASO }}$ for women with parent app $=184 \mathrm{~s}$ and $M_{\text {WASO }}$ for women without parent app $=65 \mathrm{~s}$ (see distribution Supplementary Figure S5). By comparison, the distribution of median WASO for young adult men with parent

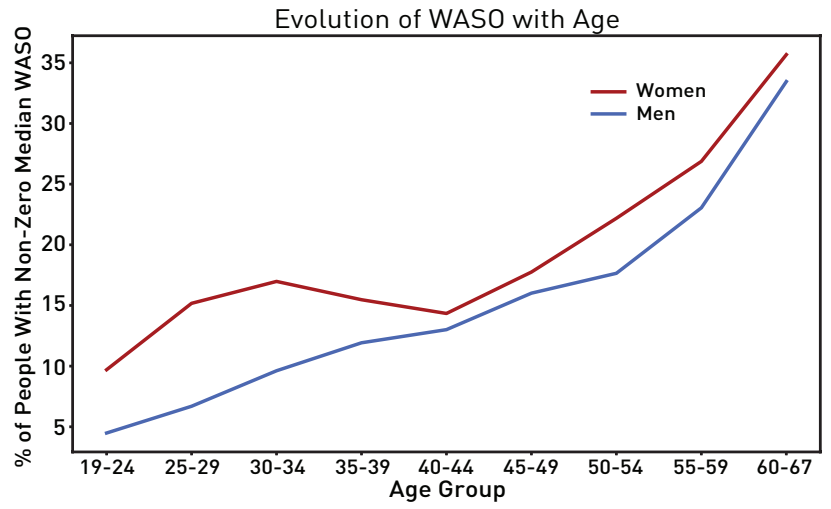

Figure 4. The percentage of people with nonzero median WASO, by age group and gender. The red color corresponds to women and blue color to men.

apps does not differ significantly from those without them (estimated with two sample KS statistics, $p=0.228$ ) where $\quad M_{\text {WASO }}$ for men with parent app $=52 \mathrm{~s}$ and $\mathrm{M}_{\text {WASO }}$ for men without parent app $=37 \mathrm{~s}$ (see distribution Supplementary Figure S5).

Next, we examine the subset of sleep observations for users with $M_{\text {WASO }} 0$, but this choice of subset eliminates the skew arising from a large fraction of users with zero WASO measurements (Supplementary Figure S5).

After applying the same comparison, we find that women aged 19-39 with parent apps installed on their phones have a significantly different distribution of mean values (denoted $\mu$ ) for their WASO than similarly aged women without parent 
apps (estimated with two-sample KS statistics, $p=1.38 * 10^{-14}$ ) where $\mu_{\text {WASO }}$ for women with parent app $=1105 \mathrm{~s}$ and $\mu_{\text {WASO }}$ for women with parent app $=874 \mathrm{~s}$ (see distribution on Supplementary Figure S6). In contrast, when we carry out the same comparison for men, we find that their distributions do not differ significantly between the group with and without parent apps (estimated with two-sample KS statistics, $p=0.207)$ and $\mu_{\text {WASO }}$ for men with parent app $=905 \mathrm{~s}$ and $\mu_{\text {WASO }}$ for men without parent app $=867 \mathrm{~s}$ (see distribution Supplementary Figure S6).

\section{Development of circadian misalignment with age}

Many people (about 75\% of the US and European labor force) maintain a conventional 5 day work week from 9 to 5 which constrains their weekly sleep behavior $[69,70]$. This recurrent temporal pattern can lead to substantial sleep deprivation during weekdays and sleep compensation during weekends, in addition to a weekend-weekday contrast in sleep timing [23]. Figure 5, D illustrates the development of weekend-weekday sleep timing differences over the lifespan (green/pink colors for onset and blue/red colors for offset). From approximately age 19
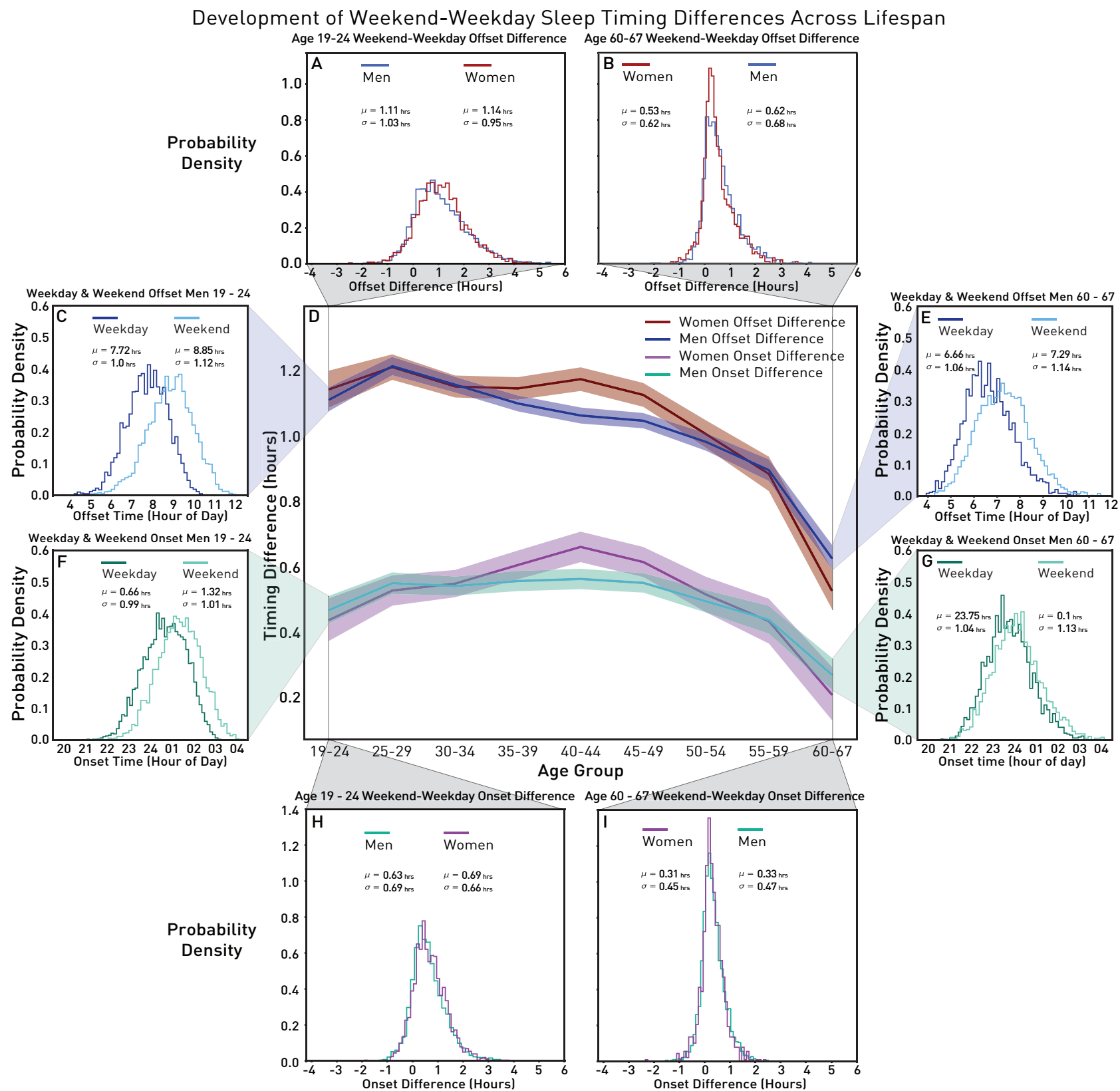

Figure 5. The development of weekend-weekday differences for sleep onset and offset by age group split by gender. The red/pink colors correspond to women, blue/ green blue colors correspond to men, darker colors represent weekend-weekday offset difference and lighter colors signify weekend-weekday onset difference. The colored envelopes display $95 \%$ CIs around each age group mean (D). The distribution of weekend-weekday differences in sleep onset for different age groups: (A) age 19-24 and (B) age 60-67. The distribution for the sleep offset weekend-weekday differences for different age groups: (H) age 19-24 and (I) age 60-67. Distribution for sleep onset time on weekdays and weekends for different age groups: (C) age 19-24 and (E) age 60-67. Distribution for sleep offset time on weekdays and weekends for different age groups: (F) age 19-24 and (G) age 60-67. 
to 55 , average sleep offset tends to be 55-70 min later on weekends while onset tends to be 25-35 min later on weekends over the same period. Thus, adults in our sample tend to sleep half an hour more on weekends than weekdays. This result is confirmed in Figure 6, B which shows the development of weekendweekday sleep duration difference with age. We identify sleep duration to be 25-40 min longer on weekends from age 19 to 55 . After age 55, weekend-weekday misalignment in onset timing declines to $20 \pm 2.6 \mathrm{~min}$ for older adult men, alongside a marginally larger decrease in offset misalignment to $38 \pm 2.3 \mathrm{~min}(95 \%$ $\mathrm{CI})$. The distributions for sleep offset and onset split by weekends and weekdays for age group 19-24 (offset Figure 5, C and onset Figure 5, F) and age group 60-67 (offset Figure 5, E and onset Figure 5, G) illustrate this contrast. For example, offset is on average $67 \pm 2.2 \mathrm{~min}$ later on weekends for men age group $19-24$ but $38 \pm 2.3$ min for men in the 60-67 year old group (95\% CI). Interestingly, average sleep offset misalignment remains greater than sleep onset misalignment into older adulthood, despite an overall convergence toward more similar weekend and weekday schedules and a reduction in weekend-weekday sleep duration difference.

Figures 5, D and 6, B indicate that misalignment in both sleep timing and duration progress similarly for men and women across the majority of the lifespan. A possible exception is visible during the 35-49 age range, during which both sleep offset and onset misalignment are slightly greater for women. Increased offset misalignment in this period for women appears to be driven by later weekend offset times compared to men, while weekday offset times are similar for both genders (see section Sleep timing and duration over the lifespan). This general similarity between genders is confirmed when observing the overlapping distribution for weekday-weekend differences of sleep onset and offset times for men and women, respectively, age group 19-24 (offset on Figure 5, A and onset on Figure 5, H) and age group 60-67 (offset Figure 5, B and onset Figure 5, I).

As before, we consider the potential biases in the data set when drawing conclusions from the figures and compare the aggregated empirical data to our mixed effects model. Our primary inferences from Figures 5 and 6 are verified by our modeling results presented in Supplementary Tables S23, S25, and S27. After controlling for country and adjusting for BMI in the mixed effects model, the slight difference between middle-aged men and women (age group 40-44, see Figure 5, D), is no longer evident or negligible due to small effect size (the model estimates men to have a $1.9 \pm 1.0 \mathrm{~min}$ higher weekend-weekday sleep offset difference and $3.0 \pm 0.8 \mathrm{~min}$ higher weekend-weekday sleep onset difference than women [95\% CI]).

\section{Sleep variability over the lifespan}

Figure 7, A and B shows the development of adult onset and offset variability with age (green/purple colors correspond to onset and blue/red to offset, while the darker shades represent weekdays and lighter shades indicate weekends). Interestingly, we find that onset variability, measured as the intra-individual standard deviation of onset time, is nearly fixed across the lifespan at $1.1 \mathrm{~h}$ on weekdays and $1.3 \mathrm{~h}$ on weekends. By comparison, offset variability decreases relatively rapidly for age group 19-24, both for men (weekdays $1.2 \pm 0.015 \mathrm{~h}$ and weekends $1.5 \pm 0.016 \mathrm{~h}$ ) and women (weekdays $1.3 \pm 0.021 \mathrm{~h}$ and weekends $1.4 \pm 0.021 \mathrm{~h}$ ) up until age 35-39, remaining around $0.9 \mathrm{~h}$ on weekdays while continuing to decrease on weekends at a gradual rate. Variability for all measurements (onset, offset, and duration) is always higher on weekends than weekdays. We find that young adults have more variable sleep offset times than onset times both on weekends and weekdays. Figure 7, C and D shows that the difference between offset variability and onset variability is positive and higher across early adulthood (19-29) for men and women on both weekends and weekdays. The weekend difference between offset and onset variability is larger for men across the age 19-34 range, while the weekday difference is larger for women in the 19-24 and 25-29 age groups.

Figure 7, F illustrates the development of sleep duration variability over the lifespan, which decreases gradually with age such that the youngest group of men have only $14 \pm 1.2 \mathrm{~min}$ higher sleep variability than the oldest group on weekends. From Figure 7, A, B, and F, we observe small significant differences between men and women; higher onset variability both on weekends and weekdays after early adulthood and consistently higher sleep offset variability on weekends for all age groups. When comparing these results to our mixed effects models which control for the influence of country and demographic covariates, we find that all of the general conclusions inferred from the descriptive plots in Figure 7 are verified (Supplementary Tables S29, S31, and S33). Taking the age 40-44 group as an example, the model estimates a 3-min higher onset variability for men than women on weekdays, 2-min greater

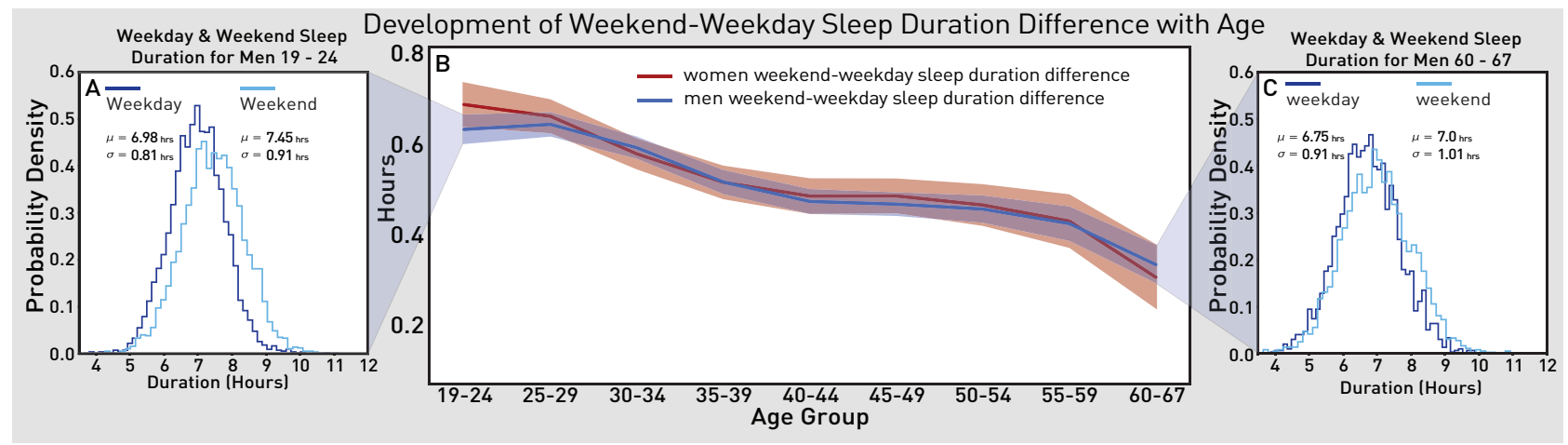

Figure 6. The development of weekend-weekday sleep duration difference by age group split up by gender. The red color corresponds to women and blue color to men. The colored envelopes display 95\% CIs around each age group mean (B). Distribution of weekend-weekday duration differences for different age groups: (A) age 19-24 and (C) age 60-67. 
Development of Sleep Variability Over the Lifespan
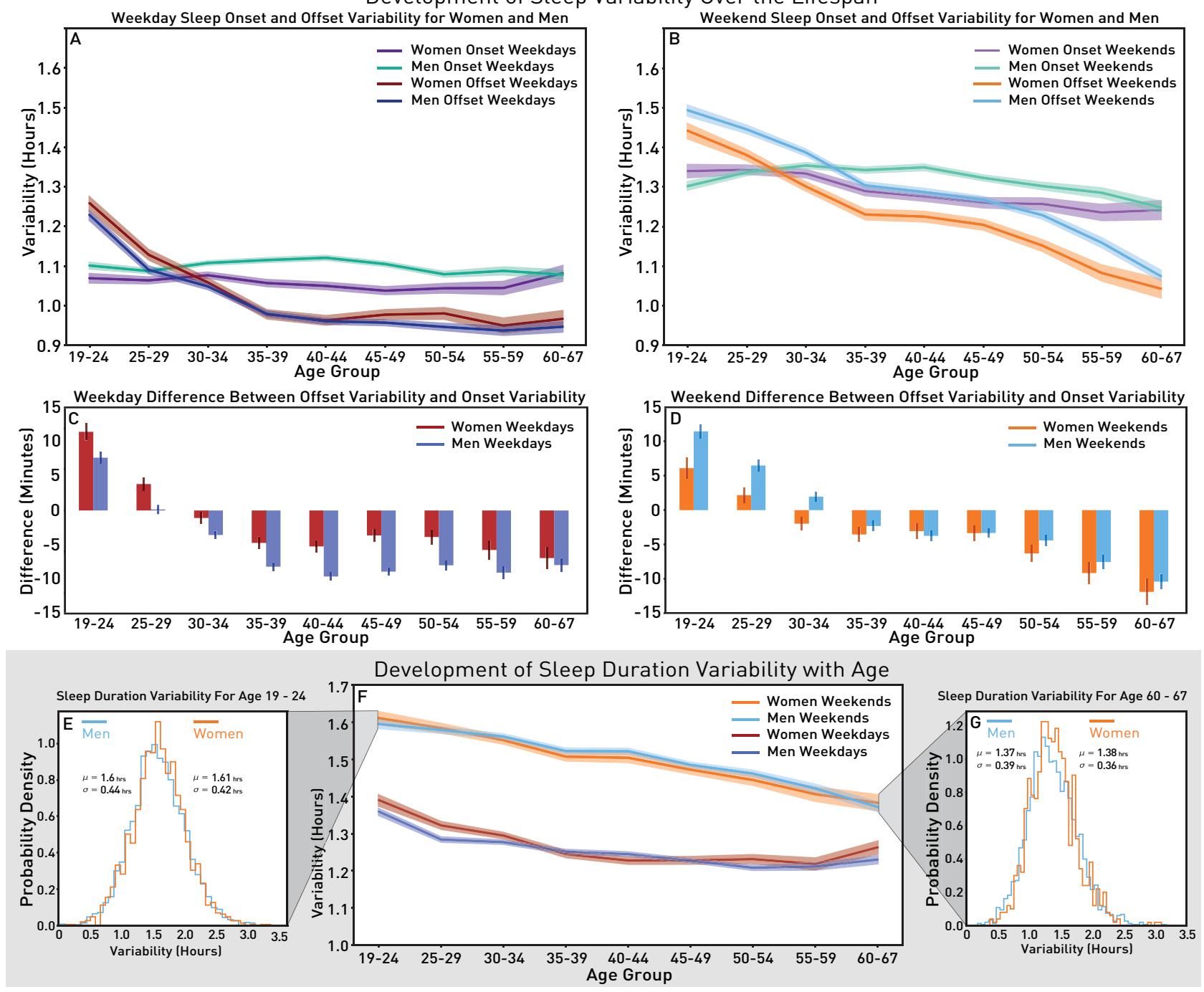

Figure 7. The development of sleep onset and offset variability with $95 \%$ CI by age group and split by gender and type of day: (A) weekdays and (B) weekends. Red/ orange curves is offset variability for women, light/dark blue is offset variability for men, light/dark purple is onset variability for women, and light/dark green is onset variability for men. Darker colors represent weekdays and lighter colors weekends. The colored envelopes display $95 \%$ CIs around each age group mean. The difference between sleep onset and offset variability with $95 \% \mathrm{CI}$ by age group on weekdays (C) and weekends (D). The development of sleep duration variability by age group split up by gender and day type (weekday/weekend). The red/orange colors correspond to women, light/dark blue colors correspond to men, darker colors represent weekdays and lighter colors signify weekends. The colored envelopes display $95 \%$ CIs around each age group mean (F). Distribution of sleep duration variability by gender for different age groups: (E) age 19-24 and (G) age 60-67.

onset variability on weekends and 5-min higher offset variability on weekends, which are trends that can also be identified on Figure 7, A and B.

\section{The effect of BMI}

In Table 3, we list average sleep onset, offset, and duration for men and women within the four BMI groups estimated with the mixed effects model (95\% CI) for age group 40-44 on weekdays. Overall, differences in sleep timing between BMI groups are statistically insignificant and/or small in effect. As one exception to this trend, we find that men within the normal BMI range sleep on average $17 \pm 2.8 \mathrm{~min}$ more than those in the obese BMI category, and men in the underweight category sleep on average $24 \pm 4.7 \mathrm{~min}$ more than those in the obese category. We carry out further discussion concerning these results in section called "BMI Discussion" in the Supplementary Information.

\section{Discussion}

Drawing on a massive global sleep dataset comprised of 11.14 million sleep observations from 69,650 adults spanning 47 countries, we confirm the presence of age-related changes in sleep duration, timing, misalignment, and variability. After controlling for baseline country-level variation using mixed effects models, we find that younger adulthood is marked by both delayed sleep onset and offset, and higher intra-individual sleep duration variability, offset variability, weekend-weekday misalignment, and weekend-weekday sleep duration difference compared with older adulthood. Conversely, sleep duration is shorter and nighttime awakenings are more prevalent during older adulthood. Only sleep onset variability exhibits little to no difference across the youngest and oldest age groups in our sample. Certain changes in sleep behavior progress consistently across most age groups observed, while others appear to be highly lifestage and/or gender dependent. In contrast to studies based on 
Table 3. Mixed effects model estimates of average sleep onset, offset, and duration for different BMI and gender groups on weekdays age 40-44 $(95 \% \mathrm{CI})$

\begin{tabular}{|c|c|c|c|c|c|c|}
\hline & \multicolumn{2}{|c|}{$\begin{array}{l}\text { Onset } \\
\text { hh:mm } \pm \mathrm{m}\end{array}$} & \multicolumn{2}{|c|}{$\begin{array}{l}\text { Offset } \\
\text { hh:mm } \pm \mathrm{m}\end{array}$} & \multicolumn{2}{|l|}{$\begin{array}{l}\text { Duration } \\
\text { hours } \pm \text { hours }\end{array}$} \\
\hline & Women & Men & Women & Men & Women & Men \\
\hline Underweight & $23: 55 \pm 8$ & $00: 10 \pm 8$ & $07: 05 \pm 7$ & $07: 11 \pm 8$ & $7.03 \pm 0.0938$ & $6.95 \pm 0.114$ \\
\hline Normal weight & $23: 45 \pm 7$ & $00: 01 \pm 7$ & $06: 58 \pm 6$ & $06: 58 \pm 6$ & $7.03 \pm 0.0938$ & $6.84 \pm 0.0952$ \\
\hline Overweight & $23: 45 \pm 7$ & $00: 01 \pm 7$ & $06: 55 \pm 6$ & $06: 55 \pm 6$ & $6.98 \pm 0.0972$ & $6.72 \pm 0.102$ \\
\hline Obese & $23: 52 \pm 8$ & $00: 10 \pm 8$ & $06: 54 \pm 6$ & $06: 54 \pm 6$ & $6.86 \pm 0.0988$ & $6.56 \pm 0.106$ \\
\hline
\end{tabular}

single or short-term observations, our unique dataset of millions of multi-night sleep recordings enables a consistent, detailed understanding of age-related tendencies in sleep patterns including intra-individual variability within inferred working and leisure periods as well as misalignment between them. We confirm several recognized age-related changes in human sleep and provide novel evidence of gender differences across key phases of adult development. Further, in the Supplementary Information, we provide (1) a comparison of our data with multiple independent large scale and global sleep datasets, (2) we explicitly compare our global estimates of social jetlag to those from Roenneberg et al. [23], and perform a quantitative exploration of underlying regional differences that appear to drive this disparity of our results compared to Roenneberg et al., and lastly, (3) we perform an exploratory analysis of the possible effect of retirement age, which varies by country and demonstrates that different regional policies appear to affect people's sleep patterns.

\section{Misalignment}

Circadian misalignment has been found to be associated with negative health outcomes such as obesity, metabolic risk factors for diabetes, and atherosclerotic cardiovascular disease, as well as adverse behaviors such as drinking and smoking which can negatively impact healthy human development [23, 71]. Notably, we find considerably lower levels of social jetlag in our sample across all observed age groups compared with the values reported by Roenneberg et al. [23] (Supplementary Figure S9). In the Roenneberg et al. [23] study, the sample consisted of questionnaire respondents from predominantly four European countries (Germany, Switzerland, the Netherlands, and Austria). Thus, the discrepancy may reflect a mismatch between global and regional circadian preferences, recall biases linked to the questionnaire and/or other unobserved differences. Constraining our sample to only include the same primary European countries as Roenneberg et al. yields markedly higher values of social jetlag across the lifespan than in our full global sample as well as altered age-related gender differences during early and late adulthood, with men incurring marginally more social jetlag than women-consistent with the age-related dependencies identified by Roenneberg et al. By contrast, in our global sample middle-aged women have marginally more social jetlag than men, with negligible gender differences in other age groups. Comparing social jetlag levels between regional strata of our sample from Asia and Europe suggests that social jetlag for young adults may be over twice as large in the same European region sampled by Roenneberg et al. [23], and $\sim 1.5$ times larger for middle-aged and older adults (Supplementary Figure S10). This provides suggestive evidence that the gap between the attenuated magnitude of social jetlag in our full sample relative to Roenneberg et al.'s may not merely be due to different means of data collection and associated measurement error (objective multi-night recording vs. self-report questionnaires). Rather, underlying regional differences appear to play an important role. We contend that accounting for underlying country-level variation is important to prevent biased global estimates of salient sleep outcomes and age-related developments.

In line with previous research, we find weekend-weekday differences in sleep timing and duration to be more pronounced among younger adults, with these elevated differences (Supplementary Figure S8) driven primarily by earlier sleep offset on weekdays and later offset on weekends than weekdays. Weekend-weekday misalignment in sleep timing and duration slightly decrease with age and decline more rapidly around the age range of 55-59, leading to near convergence of sleep onset timing on weekends and weekdays for older adults aged 60-67. Reduced misalignment in older adulthood may signal the social onset of exiting the labor force for retirement. However, some misalignment in both sleep offset, midsleep and duration persists across the age groups observed, indicating that pervasive work schedules likely continue to exert an influence on people's sleep-wake cycles through most of the adult lifespan. Our results indicate that sleep research involving adults should account for weekend-weekday heterogeneity in sleep patterns, even in older populations where nonstandard weekday schedules might otherwise be presumed.

\section{Variability}

A growing body of research indicates that irregular sleep is linked to maladaptive responses adverse to human health [25, $53,72-80]$ Outside of research on weekend-weekday misalignment, limited evidence exists about age-related changes in sleep variability in sleep patterns within individuals, particularly during weekdays and within weekends [81]. Taken together, two recent cross-sectional studies found that between-individual onset, offset, duration, and chronotype variability decrease with age [24, 45]. Similarly, a sleep diary-based study found that intraindividual variability in sleep duration decreases with age [27]. By comparison, our data set shows that intraindividual variability in sleep onset is close to fixed over the lifespanimplying that young, middle-aged, and older adults may have persistently variable sleep onset times, whereas variability in wake-up times decreases with age, likely driving the observed decline in sleep duration variability. 
We find that young adults tend to have more variable offset times than onset times, a trend which inverts after age 35 due to a decrease in sleep offset variability. One possible explanation is that a concurrent rise in weekday alarm clock use to meet fixed workplace, childcare, and/or other social commitments might drive this reduction in offset variability. Across our population, variability measurements are consistently higher on weekends, confirming that sleep patterns are more regulated on weekdays, in line with the alarm clock hypothesis. However, the gradual decline in both weekend sleep offset variability and duration variability across most of the lifespan suggests that both endogenous and exogenous factors may be involved.

\section{Nighttime awakenings}

Previous research and reviews found that women are at a greater risk than men to develop insomnia, and both insomnia and other sleep disorders are more prevalent in women during pregnancy and the postpartum period [37, 82-84]. Ours is the first study to use the contextual information encoded in app usage as a proxy for parental status to explore lifestage gender inequality in sleep quality. Importantly, the gender difference we observe in nighttime awakenings is more pronounced from young to middle adulthood than from middle to late adulthood. Supporting the hypothesis that increased sleep disturbances for women during early adulthood might be driven by childbirth and raising young children, we find a significant difference in the median WASO between women with parent apps installed on their phone and age-matched women without such applications. When we applied the same comparison to the two corresponding groups of men, we found no significant difference, a finding which suggests that the gap in prevalence of nighttime awakenings between women and men aged 19-39 may be driven by the presence of infants or young children-as well as gender-associated caretaking norms-which disproportionately interrupt the sleep of female parents. This finding is in agreement with a panel study on changes in sleep satisfaction and sleep duration after childbirth, which also found a less pronounced decrease in sleep satisfaction for men than women [85]. Others have interpreted disturbed sleep after childbirth as a contributor and/or symptom of postpartum depression [86, 87].

In line with previous observational studies that suggest age-related increases in WASO, we find that the prevalence of people regularly experiencing sleep disturbances increases with age [7, 9]. A greater proportion of women than men regularly experience some time awake after sleep onset across all age groups observed in our study, indicating that more women may have difficulty maintaining sleep even though women on average sleep longer than men. Taken together, these findings contribute to the nascent literature on the unequal burden of child rearing on women's sleep quality [88]. The use of parents apps to identify individuals with young children illustrates the promise of using contextual information related to app usage as a novel way to understand the connection between sleep and overall behavior.

\section{Sleep timing and duration}

Epidemiological studies have demonstrated that men are, on average, later chronotypes than women until 40-50 years of age, after which their circadian phase advances to overlap or become earlier [17, 24]. Our study both confirms (Supplementary Figure S7) and expands on this finding by documenting the underlying dynamics between sleep onset and offset across these age groups that shape the full sleep period and its relative position. We find that men tend to have a later sleep onset than women up until 50-54 years of age, while up until the age range of 35-39 there is no significant difference in offset time between men and women. Thereafter, from middle to late adulthood, women tend to rise later. Taken together, this inversion may be indicative of gender-gaps in both domestic and labor demands during this period from mid-late adulthood [89]. It is possible that the general overlap in wake-up times for women and men during young to middle adulthood may be due to temporarily convergent external demands characteristic of this phase of development, such as attending university, work, tending to infants and/or raising young children, etc. By choosing to focus our analysis on both the beginning and end of the sleep period, rather than just its midpoint (Supplementary Figure S7) as commonly used in epidemiological sleep studies, we capture these differences and changes which have not been consistently described before at a global scale.

The finding that men sleep less than women on average across age groups $[23,63]$, confirmed by our study, is believed to have both a biological and social basis $[89,90]$. For instance, we find that the sleep surplus for women relative to men is largest during young to middle adulthood when sleep interruptions are considerably more common for women than for men, likely due to the differential burden of caregiving. Thus, a combination of imbalanced nocturnal demands and socially imposed offset timing due to labor schedules may drive the observed gender differences in onset. Indeed, from middle to late adulthood average onset times converge and average offset times diverge. Furthermore, in line with previous research [6, 7, 9], we find that average sleep duration declines with age, with increasing portions of the average sleep distributions for both men and women falling below $7 \mathrm{~h}$ until weekday sleep duration slightly rebounds after age 60 , a phase associated with attenuated working demands due to retirement [90]. Interestingly, later weekday wake up timing in late adulthood was apparent in Germany and the United Kingdom, but was not evident for Japan within the age range of our sample. Thus regional heterogeneity in transitioning out of the labor force may be reflected by differences in the manifestation of partial sleep timing recovery (see Supplementary Information: Analysis of effective retirement age with three-way interaction of age, gender, and country). However, such recovery in sleep offset appears to be consistently more subdued for older men than women. A recent global cross-sectional study found that acute cognitive deficits in reasoning and verbal ability can arise from sleeping less than 7-8 $\mathrm{h}$ regardless of age [13]. Importantly, average weekday sleep duration for men in our sample was consistently under $7 \mathrm{~h}$ across all age groups observed.

\section{Limitations}

Several considerations should be weighed when interpreting the results of this study. First, the wearable fitness bands used rely on in-built accelerometers and proprietary algorithms developed and internally validated by a global mobile technology company. Accelerometry-based consumer sleep trackers are known to slightly overestimate sleep duration and underestimate sleep 
disruptions [66], suggesting that actual sleep duration may be slightly lower than recorded in this study and that our estimates for the prevalence of people with frequent nighttime awakenings may be conservative. Second, age and BMI were self-reported. Despite possible recall bias, we find good general agreement between the World Health Organization's country-level estimates of median age and age standardized BMI and the corresponding estimates from our data set. Nevertheless, our sampled population of wearable users may not be representative of the wider population due to potential unobserved factors also associated with wearable device ownership, such as post-secondary education attainment [91]. Third, given the cross-sectional design of the current study, we cannot statistically identify whether the observed trends across age groups correspond to withinindividual changes over the lifespan or rather reflect generational differences in normative sleep patterns. An exemplary longitudinal study which analyzed the change in diurnal timing preferences of 567 males in Finland across a 23 year period found that sleep timing shifted to become earlier with age, supporting the former intuition [92]. However, we cannot distinguish whether the age-related sleep patterns we observe are primarily driven by physiological or social developments associated with different stages of adulthood. Fourth, similar to others, we use weekends as a proxy for free days where individuals were not working to help distinguish between endogenously and exogenously driven changes $[20,24]$. This assumption does not hold for the subset of our sample who might be unemployed or otherwise follow irregular (e.g. service industry) work schedules. Thus, our estimates of misalignment may be slightly conservative. Despite these limitations, our primary results converge with recognized age-related trends in both sleep duration and timing $[9$, 28]. Furthermore, these trends appear to be generally consistent across multiple geographic regions and sociocultural contexts.

\section{Implications}

Massive data sets generated by pervasive consumer wearable devices can provide globally consistent measurements and thus can contribute unique and confirmatory insights about the development of human sleep patterns. Interestingly, the wide and overlapping distributions of sleep times between genders across the life course suggests that even though there are characteristic differences in mean values, overgeneralization of gender differences should be avoided. Underlying heterogeneity in sleep duration and timing across the life course proves the rule rather than the exception. Early weekday work schedules and norms likely constrain the varied circadian preferences of individuals, contributing to misalignment. Furthermore, given the pervasive asymmetry between weekend and weekday sleep patterns as well as variability in day-to-day sleep timing, research on behavioral interventions that promote regular sleep wake cycles is needed. Rather than impose standard morning start times, organizations might explore and evaluate person-centered work schedules and jobs that match the diverse circadian preferences of individuals, evident in this study and others.

\section{Supplementary Material}

Supplementary material is available at SLEEP online.

\section{Funding}

Sune Lehmann acknowledges funding from the Independent Research Fund Denmark (Microdynamics of Influence in Social Systems) and the Villum Foundation (Nation-scale Social Networks).

Conflict of interest statement. None declared.

\section{References}

1. Irwin MR. Why sleep is important for health: a psychoneuroimmunology perspective. Annu Rev Psychol. 2015;66:143-172.

2. Domínguez F, et al. Association of sleep duration and quality with subclinical atherosclerosis. J Am Coll Cardiol. 2019;73(2):134-144.

3. Cappuccio FP, et al. Sleep duration and all-cause mortality: a systematic review and meta-analysis of prospective studies. Sleep. 2010;33(5):585-592.

4. Vitiello MV. Sleep in normal aging. Sleep Med Clin. 2006;1(2):171-176.

5. Espiritu JRD. Aging-related sleep changes. Clin Geriatr Med. 2008;24(1):1-14.

6. LiJ,et al.Sleep in normal aging. Sleep Med Clin. 2018;13(1):1-11.

7. Floyd JA, et al. Age-related changes in initiation and maintenance of sleep: a meta-analysis. Res Nurs Health. 2000;23(2):106-117.

8. Foley D, et al. Sleep disturbances and chronic disease in older adults: results of the 2003 National Sleep Foundation Sleep in America Survey. J Psychosom Res. 2004;56(5):497-502.

9. Ohayon $\mathrm{MM}$, et al. Meta-analysis of quantitative sleep parameters from childhood to old age in healthy individuals: developing normative sleep values across the human lifespan. Sleep. 2004;27(7):1255-1273.

10. Floyd JA, et al. Changes in REM-sleep percentage over the adult lifespan. Sleep. 2007;30(7):829-836.

11. Pace-Schott EF, et al. Age-related changes in the cognitive function of sleep. Prog Brain Res. 2011;191:75-89.

12. Dijk DJ, et al. Age-related reduction in daytime sleep propensity and nocturnal slow wave sleep. Sleep. 2010;33(2):211-223.

13. Wild CJ, et al. Dissociable effects of self-reported daily sleep duration on high-level cognitive abilities. Sleep. 2018;41(12). doi: 10.1093/sleep/zsy182

14. Gadie A, et al.; Cam-CAN. How are age-related differences in sleep quality associated with health outcomes? An epidemiological investigation in a UK cohort of 2406 adults. BMJ Open. 2017;7(7):e014920.

15. Mander BA, et al. Sleep and human aging. Neuron. 2017;94(1):19-36.

16. Adan A, et al. Circadian typology: a comprehensive review. Chronobiol Int. 2012;29(9):1153-1175.

17. Roenneberg T, et al. Epidemiology of the human circadian clock. Sleep Med Rev. 2007;11(6):429-438.

18. Kantermann $\mathrm{T}$, et al. Comparing the morningnesseveningness questionnaire and munich chronotype questionnaire to the dim light melatonin onset. J Biol Rhythms. 2015;30(5):449-453.

19. Luca $\mathrm{G}$, et al. Age and gender variations of sleep in subjects without sleep disorders. Ann Med. 2015;47(6):482-491.

20. Kuula L, et al. Using big data to explore worldwide trends in objective sleep in the transition to adulthood. Sleep Med. 2019;62:69-76. 
21. Roenneberg $\mathrm{T}$, et al. Life between clocks: daily temporal patterns of human chronotypes. J Biol Rhythms. 2003;18(1):80-90.

22. Wittmann $\mathrm{M}$, et al. Social jetlag: misalignment of biological and social time. Chronobiol Int. 2006;23(1-2):497-509.

23. Roenneberg $\mathrm{T}$, et al. Social jetlag and obesity. Curr Biol. 2012;22(10):939-943.

24. Fischer D, et al. Chronotypes in the US-Influence of age and sex. PLoS One. 2017;12(6):e0178782.

25. Bei B, et al. Beyond the mean: a systematic review on the correlates of daily intraindividual variability of sleep/wake patterns. Sleep Med Rev. 2016;28:108-124.

26. Knutson $\mathrm{KL}$, et al. Intra-individual daily and yearly variability in actigraphically recorded sleep measures: the CARDIA study. Sleep. 2007;30(6):793-796.

27. Dillon HR, et al. Variability in self-reported normal sleep across the adult age span. J Gerontol B Psychol Sci Soc Sci. 2015;70(1):46-56.

28. Roenneberg $\mathrm{T}$, et al. A marker for the end of adolescence. Curr Biol. 2004;14(24):R1038-R1039.

29. Tonetti L, et al. Sex difference in sleep-time preference and sleep need: a cross-sectional survey among Italian pre-adolescents, adolescents, and adults. Chronobiol Int. 2008;25(5):745-759.

30. Bailey $M$, et al. Sex differences in circadian timing systems: implications for disease. Front Neuroendocrinol. 2014;35(1):111-139.

31. Cain SW, et al. Sex differences in phase angle of entrainment and melatonin amplitude in humans. J Biol Rhythms. 2010;25(4):288-296.

32. Duffy JF, et al. Sex difference in the near-24-hour intrinsic period of the human circadian timing system. Proc Natl Acad Sci U S A. 2011;108(Suppl 3):15602-15608.

33. Adan A, et al. Gender differences in morningnesseveningness preference. Chronobiol Int. 2002;19(4):709-720.

34. Paine SJ, et al. The epidemiology of morningness/ eveningness: influence of age, gender, ethnicity, and socioeconomic factors in adults (30-49 years).J Biol Rhythms. 2006;21(1):68-76.

35. Zimmermann LK. Chronotype and the transition to college life. Chronobiol Int. 2011;28(10):904-910.

36. Merikanto I, et al. Relation of chronotype to sleep complaints in the general Finnish population. Chronobiol Int. 2012;29(3):311-317.

37. Mallampalli MP, et al. Exploring sex and gender differences in sleep health: a Society for Women's Health Research Report. J Womens Health (Larchmt). 2014;23(7):553-562.

38. Pengo MF, et al. Sleep in women across the life span. Chest. 2018;154(1):196-206

39. Girschik J, et al. Validation of self-reported sleep against actigraphy. J Epidemiol. 2012;22(5):462-468.

40. Lauderdale DS, et al. Self-reported and measured sleep duration: how similar are they? Epidemiology. 2008;19(6):838-845.

41. Stopczynski A, et al. Measuring large-scale social networks with high resolution. PLoS One. 2014;9(4):e95978.

42. Abdullah S, et al. Towards circadian computing: early to bed and early to rise makes some of us unhealthy and sleep deprived. In: proceedings of the 2014 ACM International Joint Conference on Pervasive and Ubiquitous Computing; 2014:673-684.

43. Aledavood T, et al. Digital daily cycles of individuals. Front Phys. 2015;3:73.
44. Christensen MA, et al. Direct measurements of smartphone screen-time: relationships with demographics and sleep. PLoS One. 2016;11(11):e0165331.

45. Walch OJ,etal.A globalquantification of "normal"sleep schedules using smartphone data. Sci Adv. 2016;2(5):e1501705.

46. Cuttone A, et al. SensibleSleep: a Bayesian model for learning sleep patterns from smartphone events. PLoS One. 2017;12(1):e0169901.

47. Monsivais D, et al. Seasonal and geographical impact on human resting periods. Sci Rep. 2017;7(1):10717.

48. Fagherazzi G, et al. An international study on the determinants of poor sleep amongst 15,000 users of connected devices. J Med Internet Res. 2017;19(10):e363.

49. Roenneberg T, et al. Human activity and rest in situ. Methods Enzymol. 2015;552:257-283.

50. United Nations, Department of Economic and Social Affairs, Population Division. World Population Prospects 2019, Online Edition. Median Age of Population. 2019.

51. Ahmad OB, et al. Age standardization of rates: a new WHO standard. Geneva World Heal Organ. 2001;9(10):10.

52. WHO. Global Health Observatory Data Repository: Mean Body Max Index Trends Among Adults, Age-Standardized. Estimates by Country. Geneva, Switzerland: World Health Organization; 2019.

53. Molzof HE, et al. Intraindividual sleep variability and its association with insomnia identity and poor sleep. Sleep Med. 2018;52:58-66.

54. Consultation WHO. Obesity: Preventing and Managing the Global Epidemic. Geneva, Switzerland: WHO Technical Report Series 894; 2000.

55. WHO Expert Consultation. Appropriate body-mass index for Asian populations and its implications for policy and intervention strategies. Lancet (London, England). 2004;363(9403):157.

56. R Core Team. R: A Language and Environment for Statistical Computing. 2018. https://www.r-project.org/.

57. Faraway JJ. Extending the Linear Model with R: Generalized Linear, Mixed Effects and Nonparametric Regression Models. Boca Raton, FL: Chapman and Hall/CRC; 2016.

58. Kuznetsova A, et al. lmerTest package: tests in linear mixed effects models. J Stat Softw. 2017;82(13):2-3.

59. Luke SG. Evaluating significance in linear mixed-effects models in R. Behav Res Methods. 2017;49(4):1494-1502.

60. Depner CM, et al. Wearable technologies for developing sleep and circadian biomarkers: a summary of workshop discussions. Sleep. 2020;43(2). doi:10.1093/sleep/zsz254

61. Ancoli-Israel S, et al. The role of actigraphy in the study of sleep and circadian rhythms. Sleep. 2003;26(3):342-392.

62. Althoff $\mathrm{T}$, et al. Harnessing the web for population-scale physiological sensing: a case study of sleep and performance. In: Proceedings of the 26th International Conference on World Wide Web; 2017:113-122.

63. Ong JL, et al. Large-scale data from wearables reveal regional disparities in sleep patterns that persist across age and sex. Sci Rep. 2019;9(1):3415.

64. Lo JC, et al. Young adults' sleep duration on work days: differences between East and West. Front Neurol. 2014;5:81.

65. Shrivastava $D$, et al. How to interpret the results of a sleep study. J Community Hosp Intern Med Perspect. 2014;4(5):24983.

66. Kolla BP, et al. Consumer sleep tracking devices: a review of mechanisms, validity and utility. Expert Rev Med Devices. 2016;13(5):497-506. 
67. Martinez GM, et al. Fertility of men and women aged 15-44 in the United States: national survey of family growth, 2011-2015. Natl Health Stat Report. 2018;51(113):1-17.

68. Galland BC, et al. Normal sleep patterns in infants and children: a systematic review of observational studies. Sleep Med Rev. 2012;16(3):213-222.

69. Eurofund. Fifth European Working Conditions Survey. of the European Union PO, ed. 2012. https://www.eurofound.europa. eu/sites/default/files/ef_publication/field_ef_document/ ef1182en.pdf. Accessed July 18, 2020.

70. U.S. Bureau of Labor Statistics. Job flexibilities and work schedules summary-2017-2018. 2019. http://apps.who.int/gho/ data/view.main.CTRY12461?lang=en. Accessed July 1, 2020.

71. Wong PM, et al. Socialjetlag, chronotype, and cardiometabolic Risk. J Clin Endocrinol Metab. 2015;100(12):4612-4620.

72. Gooley JJ. How much day-to-day variability in sleep timing is unhealthy? Sleep. 2016;39(2):269-270.

73. Lemola $S$, et al. Variability of sleep duration is related to subjective sleep quality and subjective well-being: an actigraphy study. PLoS One. 2013;8(8):e71292.

74. Telzer EH, et al. Sleep variability in adolescence is associated with altered brain development. Dev Cogn Neurosci. 2015;14:16-22

75. Becker SP, et al. Intraindividual variability of sleep/wake patterns in relation to child and adolescent functioning: a systematic review. Sleep Med Rev. 2017;34:94-121.

76. Patel SR, et al.; Osteoporotic Fractures in Men (MrOS); Study of Osteoporotic Fractures (SOF) Research Groups. The association between sleep patterns and obesity in older adults. Int J Obes (Lond). 2014;38(9):1159-1164.

77. Okun ML, et al. Sleep variability, health-related practices, and inflammatory markers in a community dwelling sample of older adults. Psychosom Med. 2011;73(2):142-150.

78. Veeramachaneni $\mathrm{K}$, et al. Intraindividual variability in sleep and perceived stress in young adults. Sleep Health. 2019;5(6):572-579.

79. Slavish DC, et al. Intraindividual variability in sleep and comorbid medical and mental health conditions. Sleep. 2019;42(6). doi:10.1093/sleep/zsz052
80. Murray JM, et al.; Delayed Sleep on Melatonin (DelSoM) Study Group. Sleep regularity is associated with sleepwake and circadian timing, and mediates daytime function in delayed sleep-wake phase disorder. Sleep Med. 2019;58:93-101.

81. Taylor BJ, et al. Bedtime variability and metabolic health in midlife women: the SWAN sleep study. Sleep. 2016;39(2):457-465.

82. Moline ML, et al. Sleep in women across the life cycle from adulthood through menopause. Sleep Med Rev. 2003;7(2):155-177.

83. Zhang $B$, et al. Sex differences in insomnia: a meta-analysis. Sleep. 2006;29(1):85-93.

84. Kendall-Tackett KA. Depression in New Mothers: Causes, Consequences and Treatment Alternatives. Oxfordshire, UK: Routledge; 2016.

85. Richter $\mathrm{D}$, et al. Long-term effects of pregnancy and childbirth on sleep satisfaction and duration of first-time and experienced mothers and fathers. Sleep. 2019;42:5-7.

86. Newport DJ, et al. The treatment of postpartum depression: minimizing infant exposures. J Clin Psychiatry. 2002;63(Suppl 7):31-44

87. Goyal D, et al. Fragmented maternal sleep is more strongly correlated with depressive symptoms than infant temperament at three months postpartum. Arch Womens Ment Health. 2009;12(4):229-237.

88. Venn S, et al. The fourth shift: exploring the gendered nature of sleep disruption among couples with children. $\mathrm{Br} J$ Sociol. 2008;59(1):79-97.

89. Burgard SA, et al. Gender and time for sleep among U.S. adults. Am Sociol Rev. 2013;78(1):51-69.

90. Hagen EW, et al. Changes in sleep duration and sleep timing associated with retirement transitions. Sleep. 2016;39(3):665-673.

91. Macridis S, et al. Consumer physical activity tracking device ownership and use among a population-based sample of adults. PLoS One. 2018;13(1):e0189298.

92. Broms $\mathrm{U}$, et al. Long-term consistency of diurnal-type preferences among men. Chronobiol Int. 2014;31(2):182-188. 